\title{
Spatial S-R compatibility effects with unimanual two-finger choice reactions for prone and supine hand positions
}

\author{
GABRIELE HEISTER \\ Universität Tübingen, Tübingen, W. Germany \\ WALTER H. EHRENSTEIN \\ Institut für Arbeitsphysiologie, Dortmund, W. Germany \\ and \\ PETER SCHROEDER-HEISTER \\ Universität Konstanz, Konstanz, W. Germany
}

\begin{abstract}
The influence of spatial stimulus-response (S-R) compatibility on unimanual two-finger choice reactions was investigated. Subjects had to react as fast as possible to a flash of light that was presented in the right or left visual field. They used the index or middle fingers of their left or right hands to press the spatially same (compatible) key or the spatially different (incompatible) key. In Condition 1 the subjects' palms faced down (pronation); in Condition 2 their palms faced up (supination) so that the spatial order of the fingers was reversed. A strong compatibility effect between right and left field of stimulation and spatially right and left finger was found under both conditions. Compatible reaction times were shorter than incompatible reaction times by $52 \mathrm{msec}$ in the palm-down condition and by $61 \mathrm{msec}$ in the palm-up condition. The results are interpreted as supporting the coding hypothesis of spatial compatibility.
\end{abstract}

As has been shown repeatedly, choice reaction times to stimuli are shorter when stimuli and responses are compatible with respect to perceptual or symbolic features than when they are incompatible: Within such paradigms the present study is concerned with spatial stimulus-response (S-R) compatibility, with stimulus and response positions corresponding in the spatial dimension right versus left. Usually, two types of spatial S-R compatibility effects are distinguished: those with relevant stimulus location, and those with irrelevant stimulus location. In the relevant type, subjects are concerned with the spatial position of the stimulus (they press, for example, a key on the right side if a light occurs in the right visual field, and a key on the left if it occurs on the left). In the irrelevant type, they have to make decisions based on some nonlocational feature of the stimulus (e.g., its shape or color), but its spatial position is nevertheless effective (e.g., a reaction

\footnotetext{
The research reported here was carried out at the Institut fur Arbeitsphysiologie, Dortmund. The results were presented in part at the 28th Tagung experimentell arbeitender Psychologen, Saarbrücken, March 23-27, 1986. We would like to thank Dirk Vorberg for stimulating suggestions, Armin Bozek and Wilfried Runte for technical assistance, the reviewers for helpful comments, and David Emmans for revising the English text.

G. Heister is with the Psychologisches Institut, Universität Tübingen, and P. Schroeder-Heister is with the Fachgruppe Philosophie, Universität Konstanz. Requests for reprints should be addressed to W. $\mathbf{H}$. Ehrenstein, Institut für Arbeitsphysiologie, Ardeystrasse 67, 4600 Dortmund 1, W. Germany.
}

with a right key is faster to a stimulus in the right field than to a stimulus in the left field, and so on).

Spatial S-R compatibility ${ }^{1}$ may be best explained by the coding hypothesis. According to this hypothesis, the positions of stimuli and responses are represented and compared in a spatial code, implying that incompatible $S-R$ pairings require additional effort of translation and thus lead to longer reaction times. The coding hypothesis was first proposed by Fitts and Seeger (1953) and has since been further developed, especially by Wallace (1971), Nicoletti, Anzola, Luppino, Rizzolatti, and Umiltà (1982), and Umiltà and Nicoletti (1985). Although the concept of coding is still rather unclear, in most cases investigated hitherto the coding hypothesis has been superior to alternative theories, such as (1) the thesis that spatial compatibility is related to neuroanatomical connectivity, (i.e., that it is a pathway effect); (2) the idea of an implicit mapping of spatial relations onto parts of the body (for example, a natural association of the anatomically right and left hands as spatially right and left); and (3) attentional theories, according to which spatial compatibility is based on a tendency to react toward the stimulus source.

Theories of the first type (neuroanatomical pathway hypotheses) consider the reaction time advantage for compatible $S-R$ associations to be caused by the directness of neuroanatomical pathways in the following sense: The ipsilateral relation between side of stimulation and responding hand (e.g., right-hand reactions to right-field stimuli) 
requires no passing of information between the hemispheres, provided the information is processed in the hemisphere where it directly arrives and the reaction is controlled solely by the hemisphere opposite to the responding hand. In the case of a contralateral reaction (e.g., right-hand reaction to a left-field stimulus), the hemisphere where the signal arrives and the one that controls the responding hand are different, so that a callosal crossing between hemispheres is necessary, which provokes a delay of the response. Apart from the fact that this theory would explain only an effect in the amount of one callosal crossing time, an effect considerably smaller than compatibility effects (see Bashore, 1981), such an explanation has been ruled out as a determining factor of spatial compatibility by studies in which subjects had to respond with crossed arms (see Wallace, 1971). Wallace was able to show that the S-R compatibility effect is tied to the spatial position of the arm and not to the anatomical (right or left) arm used for response, as would have to be expected by the neuroanatomic theory. Another argument against the pathway hypothesis is that spatial compatibility effects are also obtained along the above/below dimension (for irrelevant stimulus location see Hedge \& Marsh, 1975; for relevant stimulus location, see Nicoletti \& Umiltà, 1984, 1985, who also used the crossed-hands technique). In this case, a neuroanatomic correlation is not so apparent as it is for the right/left dimension. Strictly speaking, the pathway hypothesis should be treated distinctly within the field of S-R compatibility, inasmuch as the term $S-R$ compatibility effect is usually applied to denote an observed reaction time advantage for compatible S-R pairings which cannot be accounted for on neuroanatomical grounds (see Heister \& SchroederHeister, 1985). Nevertheless, one has to consider that a small pathway effect can be expressed in reaction time data and may add to "genuine"' S-R compatibility effects (see, e.g., Berlucchi, Crea, Di Stefano, \& Tassinari, 1977).

Theories of the second type, which we call mapping hypotheses, are based on the assumption that spatial relationships are naturally mapped onto parts of the body. Such a mapping, for example, would be the natural association of the anatomically right and left hands as spatially right and left. Likewise, the index and middle fingers of the right hand and the middle and index fingers of the left hand would be associated as spatially left and right. The assumption behind such a theory is that, if the body is in some "normal" position, certain anatomical differences (e.g., right and left hands) are actually combined with corresponding spatial distinctions. For example, in a normal position the anatomically right hand is on the spatially right side of the body midline, and the left hand is on the left side. It is then assumed that this association, which is present in normal body positions, is maintained if the body is in some non-normal position, for example, if the hands are crossed. Experiments using the crossedhands design have shown that such a mapping is not the determining factor for spatial compatibility, that is, that spatial compatibility rests upon compatibility between stimulus positions and response positions and not between stimulus positions and mapping onto response organs (see, e.g., Brebner, Shephard, \& Cairney, 1972). Therefore, since for the "standard" spatial compatibility design the crossed-hands test is evidence against the mapping hypothesis and in favor of the coding hypothesis, the mapping hypothesis has never been used to explain spatial compatibility in general.

Nevertheless, even if such mapping is not effective for the task required in studies of spatial compatibility, some authors assume that it is present and effective, namely in being responsible for the overall larger reaction times in the crossed-hands conditions. It is hypothesized that crossing the hands leads to an incongruency between mapped spatial positions and actual spatial positions of the hands with respect to each other (Brebner et al., 1972; Nicoletti, Umiltà, \& Ladavas, 1984). Furthermore, Ladavas and Moscovitch (1984) used the mapping hypothesis to explain a result that was an exception to the normal S-R pattern. Under an experimental condition in which subjects had to react with their heads tilted to the right or left, they obtained a reversal of the spatial compatibility effect with hands crossed. (For an alternative interpretation of Ladavas \& Moscovitch's result, not employing the mapping hypothesis, see Heister \& Schroeder-Heister, 1986). Therefore, the mapping hypothesis may be considered a possible alternative to the coding hypothesis in special applications.

Theories of the third type (i.e., attentional hypotheses; the term originated in Nicoletti et al., 1982) were favored in particular by Simon (e.g., Simon, 1969; Simon \& Rudell, 1967). According to this concept, spatial S-R compatibility effects result, for example (Simon, 1969, p. 174), from a " 'natural' tendency to respond toward the source of stimulation" (orienting reflex). However, a spatial compatibility effect can be demonstrated when both stimuli are on one side of the body midline and the response keys are on both sides, and even when both the stimuli and the response keys are on one side of the body midline (for relevant stimulus location, see Nicoletti et al., 1982; for irrelevant stimulus location, see Umiltà \& Nicoletti, 1985). These findings show that it is the relative locations of stimuli and responses that are effective for spatial compatibility, not their locations with respect to the body midline, as would be required by Simon's attentional explanation. One should concede, however, that the attentional hypothesis has mainly been formulated for S-R compatibility effects with irrelevant stimulus location. (For a detailed discussion of attentional vs. coding hypotheses, see Umiltà \& Nicoletti, 1985.)

In the present study spatial compatibility was investigated under conditions of relevant stimulus location for unimanual two-finger choice reactions, in order to test whether a spatial compatibility effect known for hands also takes place for fingers. As a substitute for the crossedhands technique, which has proved to be so efficacious in investigations of bimanual choice reactions, we chose a design in which the hands were held with the palms up 
or down. A supine position leads to a reversal of the spatial order of the fingers, so that we can distinguish between effects due to spatial and anatomical position of the fingers. Bradshaw and Perriment (1970) investigated unimanual two-finger choice reactions with hands held in prone and supine positions, but they studied only the aspect of laterality effects. They held spatial compatibility constant throughout their experiment; that is, their subjects always responded under a compatible S-R condition. Thus, the present experiments extended their technique to the investigation of spatial compatibility. In another context, Miller (1982) and Reeve and Proctor (1985; see also further references therein) worked with four-finger responses (two fingers of each hand), but again with spatial compatibility for fingers held constant.

Katz (1981) obtained a spatial compatibility effect for unimanual two-finger choice reactions, but with hands held prone in the body midline. Apart from also investigating the supine position, we had the subjects react with the responding hand in the normal right or left position, that is, the right hand on the right and the left hand on the left side of the body midline. This design allowed us to discriminate more easily between attentional and coding explanations (see below). Another difference between our design and Katz's was that Katz used irrelevant stimulus location.

Unlike Nicoletti et al. (1982) in their investigation of bimanual reactions on the same side of the body midline, we decided to present the stimuli in the right and left visual fields instead of on one side of the body midline. This enabled us to test whether a spatial compatibility effect is present not only between (right or left) field of stimulation and responding (right or left) finger but also between field of stimulation and responding (right or left) hand.

The different theories mentioned above, namely the coding hypothesis and, as its rivals, the neuroanatomical pathway hypothesis, the mapping hypothesis, and the attentional hypothesis, lead to different predictions for our experiment. According to the coding hypothesis, a spatial compatibility effect should be obtained between fingers in the palm-down position, despite the fact that the hands are held on the right or left so that the two responding fingers are never on different sides of the body midline. This is because the relative positions of stimuli and responses are effective in this case. Also, this S-R compatibility effect for fingers should remain when the hands are held palm up, since the spatial and not the anatomical relationships between fingers are supposed to be encoded. Finally, a spatial compatibility effect with respect to responding hands should not occur. The coding hypothesis predicts no such additional effect for hands because for each response the hand to be used is fixed in advance, so that only the position of a single hand with respect to the body is involved, whereas the relative spatial difference between the two responding hands should not be important.
The neuroanatomical pathway hypothesis predicts no reaction time difference between fingers, in either the prone or the supine position, since the reactions for both fingers of one hand are initiated in the same hemisphere. It would predict only a small reaction time advantage for compatible hand-stimulus pairings in the amount of one callosal crossing time.

The mapping hypothesis postulates that the spatially right and left positions are naturally mapped onto the middle and index fingers of the (anatomically) right hand and onto the index and middle fingers of the left hand. Thus, since compatibility is now bound to the anatomical fingers instead of to their positions, an S-R compatibility effect would be expected for the prone condition, whereas a reversal of this effect should occur for the supine condition, since the spatial order of the anatomical fingers is reversed. If one also assumes a mapping of spatially right and left positions onto right and left hands, an effect similar to a spatial S-R compatibility effect is predicted between responding hands.

The attentional hypothesis predicts a compatibility effect for hands (because they are on the right and left sides of the body midline) but not for the responding fingers (because both fingers are either on the right or on the left), regardless of whether the hands are in prone or supine positions. The attentional hypothesis, at least in its standard form, is not sensitive to relative spatial locations on the same side of the body midline but only to locations with respect to the body midline.

\section{METHOD}

\section{Subjects}

Eight female college students (aged 17-18 years) served as paid subjects. All were right-handed according to a German adaptation of the Edinburgh Inventory (Oldfield, 1971), had normal vision, and were naive as to the purpose of the task. All subjects served in both experimental conditions.

\section{Apparatus}

Subjects sat in front of a modified Förster perimeter (OCULUS). Each subject's head position was fixed by a forehead-and-chin rest; the distance between the subject's eyes and the perimeter plane was $45 \mathrm{~cm}$. Two shielded lamps provided a dim and diffuse ambient illumination. The stimuli were presented by two red light-emitting diodes (LEDs; Hewlett-Packard subminiature HLMP-6620). The circular LED lights subtended $10^{\prime}$ of arc. They were positioned at $5^{\circ}$ of visual angle to the left and right of the fixation point. The fixation point consisted of a white circular field subtending a visual angle of $0.75^{\circ}$ on a gray perimeter plane. Luminance (measured by a Hagner Universal photometer S2) was $0.42 \mathrm{~cd} / \mathrm{m}^{2}$ for the perimeter background, $1.2 \mathrm{~cd} / \mathrm{m}^{2}$ for the fixation point, and $81 \mathrm{~cd} / \mathrm{m}^{2}$ for the LED lights. The subjects' ability to maintain fixation properly was tested in pretrials in which eye movements were monitored by an infrared photoelectric device displayed on an oscilloscope. An acoustic warning $150 \mathrm{msec}$ in duration preceded the stimulus onset by 500 to $800 \mathrm{msec}$. The stimuli were presented for $100 \mathrm{msec}$. Subjects had to press one of two keys on a microswitch box with their index or middle fingers as fast as possible while maintaining their gaze on the fixation point. The microswitches (Schadow-Digitast SE, with electronic rebound suppression) were 
connected to an electronic clock that was started with the stimulus onset and stopped by the microswitch contact. The microswitch box was freely movable and was attached either to the left or to the right side of the experimental desk (for the prone condition) or it was fixed underneath a shelf placed on the desk, allowing the hands to press the key from below (for the supine condition).

\section{Procedure}

The subjects attended two sessions on different days: In one session the subjects responded with the palms face down (pronation), in the other session with the palms face up (supination). Half of the subjects started with the palm-down condition and the other half with the palm-up condition. Each session was subdivided into eight blocks, separated by short rest periods. Each block consisted of 6 practice trials and 44 test trials. Stimuli were presented in a particular quasi-random order within each block, allowing a maximum of only three consecutive presentations of the same (right or left) stimulus. In four blocks of each session the subjects made compatible responses, that is, a right-finger response to right lights and a left-finger response to left lights (both for right and for left hands). In the other four blocks they made incompatible responses, that is, a right-finger response to left lights and a left-finger response to right lights (again, both for right and for left hands). To prevent possible cognitive/motor interference in the hemisphere controlling the responding hand, which may be obtained when only one hand is used for a long period (see Heister, 1984), the responding hand was altered from one block to the next. For all subjects, each session consisted of two halves, which were identical with respect to the order of blocks. Each subject obtained a different order of blocks, which was balanced according to a Latin square design. For each block, the subjects were told to use the appropriate finger (index or middle finger), in order not to draw their attention to the distinction between compatible and incompatible conditions. Errors were few, and error trials were repeated at the end of each block.

\section{RESULTS}

Median reaction times were computed for right- and left-hand responses to right- and left-field stimulation for compatible and incompatible light-finger relations; that is, for each subject, eight values were obtained in Condition 1 (prone hand position) and eight in Condition 2 (supine hand position). These median reaction times of the responses were subjected to a four-way within-subjects analysis of variance with the following factors: experimental condition (hands held in pronation or supination), field of stimulus presentation (right or left), reacting hand (right or left), and reacting finger (right or left). The corresponding cell means and standard deviations are given in Table 1.
There were two significant main effects: Reactions with hands held prone were $17 \mathrm{msec}$ faster than reactions with hands held supine $[297$ vs. $314 \mathrm{msec}, F(1,7)=6.42$, $p=.039$ ], and reactions with right-field stimulation were $14 \mathrm{msec}$ faster than reactions with left-field stimulation [298 vs. $312 \mathrm{msec}, F(1,7)=20.44, p=.0027]$. For the present study it was of greater interest whether an interaction occurs between field of stimulation and responding finger, which expresses the spatial compatibility effect for fingers. This interaction was in fact highly significant $[F(1,7)=61.84, p=.0001]$. Compatible reactions, that is, reactions with right fingers to right lights and left fingers to left lights, were $\mathbf{5 2} \mathrm{msec}$ faster for hands held in pronation and $61 \mathrm{msec}$ faster for hands held in supination than were incompatible reactions, that is, reactions with right fingers to left lights and with left fingers to right lights (271 vs. $323 \mathrm{msec}$ and 284 vs. $345 \mathrm{msec}$, respectively; see Figures 1 and 2). The interaction between responding hand and field of stimulation, which would express a compatibility effect for hands, was not significant $[F(1,7)=1.79, p=.2232]$.

As Figures 1 and 2 illustrate, the spatial compatibility effect for fingers did not shift from the palm-down to the palm-up condition: The spatially right finger was always faster with right-field stimulation than with left-field stimulation (pronation, 270 vs. $334 \mathrm{msec}$; supination, 275 vs. $352 \mathrm{msec}$ ), and the spatially left finger was faster with left-field stimulation than with right-field stimulation (pronation, 271 vs. $312 \mathrm{msec}$; supination, 292 vs. $337 \mathrm{msec}$ ). Correspondingly, with right-field stimulation the right finger was always faster than the left finger (pronation, 270 vs. $312 \mathrm{msec}$; supination, 275 vs. $337 \mathrm{msec}$ ), and with left-field stimulation the left finger was always faster than the right finger (pronation, 271 vs. $334 \mathrm{msec}$; supination, 292 vs. $352 \mathrm{msec}$ ).

The interaction between experimental condition, field, and finger (which would indicate a reversal of the spatial compatibility effect) was not significant $[F(1,7)=3.60$, $p=.0995]$. Separate analyses of variance for the palmdown and palm-up conditions showed that the interaction between field of stimulation and responding finger was highly significant in both cases [palm-down, $F(1,7)=$ $52.13, p=.0002 ;$ palm-up, $F(1,7)=59.18$, $p=.0001]$, but the S-R compatibility effect tended to be somewhat larger for hands held palm up.

Table 1

Means of the Median Reaction Times (in Milliseconds) and Standard Deviations (in Parentheses)

\begin{tabular}{|c|c|c|c|c|c|c|c|c|}
\hline \multirow[b]{3}{*}{ Condition } & \multicolumn{4}{|c|}{ Left Light } & \multicolumn{4}{|c|}{ Right Light } \\
\hline & \multicolumn{2}{|c|}{ Left Hand } & \multicolumn{2}{|c|}{ Right Hand } & \multicolumn{2}{|c|}{ Left Hand } & \multicolumn{2}{|c|}{ Right Hand } \\
\hline & $\begin{array}{c}\text { Left } \\
\text { Finger }\end{array}$ & $\begin{array}{l}\text { Right } \\
\text { Finger }\end{array}$ & $\begin{array}{c}\text { Left } \\
\text { Finger }\end{array}$ & $\begin{array}{c}\text { Right } \\
\text { Finger }\end{array}$ & $\begin{array}{c}\text { Left } \\
\text { Finger }\end{array}$ & $\begin{array}{l}\text { Right } \\
\text { Finger }\end{array}$ & $\begin{array}{c}\text { Left } \\
\text { Finger }\end{array}$ & $\begin{array}{c}\text { Right } \\
\text { Finger }\end{array}$ \\
\hline $\begin{array}{c}1 \\
\text { (Prone Hand) }\end{array}$ & $\begin{array}{l}275 \\
\text { (19) } \\
\text { MF }\end{array}$ & $\begin{array}{l}343 \\
(33) \\
\text { IF }\end{array}$ & $\begin{array}{l}268 \\
(32) \\
\text { IF }\end{array}$ & $\begin{array}{l}324 \\
(28) \\
\text { MF }\end{array}$ & $\begin{array}{l}323 \\
(52) \\
\mathrm{MF}\end{array}$ & $\begin{array}{c}264 \\
\text { (26) } \\
\text { IF }\end{array}$ & $\begin{array}{l}301 \\
(39) \\
\text { IF }\end{array}$ & $\begin{array}{l}276 \\
(25) \\
\text { MF }\end{array}$ \\
\hline $\begin{array}{c}2 \\
\text { (Supine Hand) }\end{array}$ & $\begin{array}{l}284 \\
(20) \\
\text { IF }\end{array}$ & $\begin{array}{l}363 \\
(32) \\
\text { MF }\end{array}$ & $\begin{array}{l}301 \\
(35) \\
\text { MF }\end{array}$ & $\begin{array}{l}342 \\
(39) \\
\text { IF }\end{array}$ & $\begin{array}{c}323 \\
(40) \\
\text { IF }\end{array}$ & $\begin{array}{l}279 \\
(22) \\
\text { MF }\end{array}$ & $\begin{array}{l}351 \\
(43) \\
\text { MF } \\
\end{array}$ & $\begin{array}{l}270 \\
(28) \\
\text { IF }\end{array}$ \\
\hline
\end{tabular}

IF = index finger; $M F=$ middle finger. 


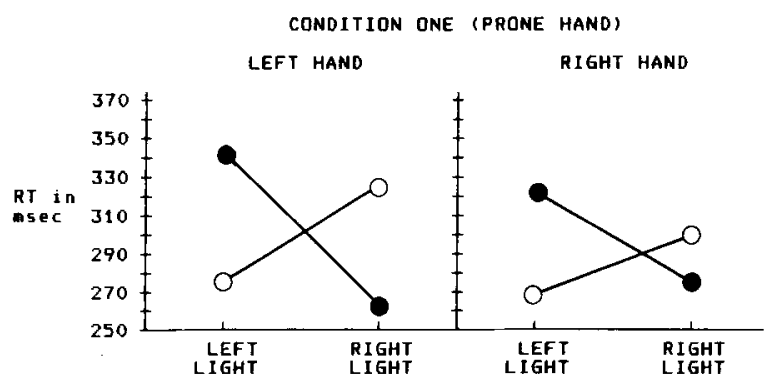

Figure 1. Means of the median reaction times for responses with the right finger (filled circles) or left finger (unfilled circles) of the right or left hand to lights in the right or left visual field under experimental Condition 1 (hands held in pronation).

CONDITION TWO (SUPINE HAND)

LEFT HAND

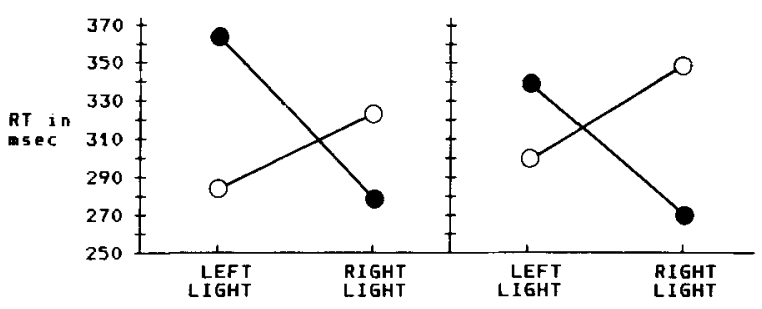

Figure 2. Means of the median reaction times for responses with the right finger (filled circles) or left finger (unfilled circles) of the right or left hand to lights in the right or left visual field under experimental Condition 2 (hands held in supination).

The interaction between experimental condition, responding hand, and responding finger was significant $[F(1,7)=12.86, p=.0089]$. This may express anatomical finger differences, since turning the hands leads to a reversal of right and left fingers. For example, the right finger of the right hand with the palm held down is the middle finger, and the left finger is the index finger; with the palm held up, the right finger of the right hand is the index finger, and the left finger is the middle finger. In order to clarify this aspect, separate two-way analyses of variance were performed for the palm-down and palmup conditions (factors: hand and anatomical, i.e., middle or index, finger), for the right and left hands (factors: experimental condition and anatomical finger), and index and middle fingers (factors: experimental condition and hand), right and left field always being combined. A significant interaction between hand and anatomical finger for the palm-down condition $[F(1,7)=6.89, p=.034]$ indicated that the index finger of the right hand reacted much faster than the other fingers ( 284 vs. 304,300 , and $299 \mathrm{msec}$ ). For the palm-up condition, there was a significant main effect for anatomical fingers $[F(1,7)=$ $12.72, p=.009$ ], indicating that, overall, middle fingers reacted more slowly than did index fingers ( $323 \mathrm{vs.}$ $305 \mathrm{msec}$ ). The analysis for right-hand reactions showed a significant main effect for experimental condition $[F(1,7)$ $=7.21, p=.031]$, which indicates that reactions were slower with hands held palm up (316 vs. $292 \mathrm{msec}$ ). A significant main effect for anatomical fingers in the same analysis $[F(1,7)=10.91, p=.013]$ indicates that for right-hand reactions there was a reliable difference between index and middle fingers ( $295 \mathrm{vs.} 313 \mathrm{msec}$ ) that was not present for left-hand reactions [304 vs. $310 \mathrm{msec}$; $F(1,7)=3.95$, n.s.]. For left-hand reactions, the interaction between experimental condition and anatomical finger was significant $[F(1,7)=16.94, p=.0045]$, which indicates that the index finger of the left hand was equally fast in the palm-down and palm-up conditions (304 $\mathrm{msec}$ ), whereas the middle finger was much slower in the palm-up than in the palm-down condition (321 vs. $299 \mathrm{msec}$ ). The analyses for index and middle fingers showed that only the middle fingers were significantly slower under the palm-up than under the palm-down condition [index fingers; 305 vs. $294 \mathrm{msec}, F(1,7)=3.09$, n.s.; middle fingers; 324 vs. $299 \mathrm{msec}, F(1,7)=7.17$, $p=.032]$. Altogether, the three-way interaction of the grand ANOVA demonstrates an anatomical finger difference in the sense that the index finger of the right hand was fastest and that turning the hand palm up influenced the reactions of the different fingers differently. These anatomical finger effects do not affect the results for spatial compatibility, since the reaction times relevant for this effect are composed of the reaction times of index and middle fingers.

Finally, an interaction was obtained between experimental condition, field of stimulation, responding hand, and responding (right or left) finger $[F(1,7)=$ $27.82, p=.0012]$. This four-way interaction indicates that for right-hand reactions in the palm-down condition, the difference between incompatible and compatible finger was smaller with right-field stimulation (where it was left minus right finger) than with left-field stimulation (where it was right minus left finger) and that this was reversed in the palm-up condition. Left-hand reactions showed a tendency to the opposite pattern. (In Figures 1 and 2, the differences for incompatible minus compatible finger are represented as the distances between values displayed one above the other.) This was confirmed through a post hoc two-way analysis of variance with the factors experimental condition (prone vs. supine position) and field-hand relationship (compatible vs. incompatible pairing) for the finger-compatibility effect, that is, for the difference scores of incompatible and compatible fingers. This analysis showed that the finger-compatibility effect depended on whether the responding hand was compatible with the field of stimulation. In the prone position the fingercompatibility effect was smaller overall with compatible hand-field relations and larger with incompatible ones, and in the supine position the reverse was true, as was shown by a significant interaction between experimental condition and hand-fieid relationship $[F(1,7)=12.84$, $p=.0089]$. This unexpected result might express a more complicated kind of S-R compatibility effect than the one that was under investigation here. It may be called a second-order S-R compatibility effect, in the sense that only the amount, not the direction (positive or negative), 
of the difference between incompatible and compatible fingers is affected by the compatibility of responding hand and side of stimulation.

No other main effects or interactions of the grand ANOVA reached significance.

\section{DISCUSSION}

The main question addressed by this study was whether a spatial S-R compatibility effect occurs with two-finger choice reactions for hands held in the prone position, and whether this effect persists when the palms are turned upward. The results show a clear-cut compatibility effect for fingers under both conditions, which was even more pronounced with hands held in supination (see Figures 1 and 2).

This effect is in accordance with the predictions of the coding hypothesis of spatial S-R compatibility, which says that the relative spatial positions of stimuli and responses are encoded and compared, and thus produce an effect irrespective of the anatomical posture of the response organs. The neuroanatomical pathway hypothesis is immediately refuted, since it would not even predict different reaction times for compatible versus incompatible S-R pairings in the prone hand position, as the responses of both fingers of one hand are initiated in the same hemisphere. The mapping hypothesis, postulating a natural association of right and left positions with anatomically right and left fingers, is ruled out because it predicts a reversal of the effect with the hand held supine, contrary to our result.

Nevertheless, the mapping hypothesis may explain the result that in the supine condition reactions were generally slower, which agrees with analogous results for the crossed-hands test with bimanual reactions (see Nicoletti et al., 1984). Mapping would then be present, but it would be effective not for spatial compatibility but only for the overall speed of response under each of the two conditions. For hands held with the palms up, there would be an incongruity between relative spatial positions of fingers (for the right hand, index finger on the right side, middle finger on the left side; for the left hand, vice versa) and the spatial positions naturally mapped onto the fingers (for the right hand, index finger = left, middle finger = right; for the left hand, vice versa), which would cause the relative slowness under the palm-up condition.

However, the assumption of some mapping of right and left positions onto the anatomical fingers, even if it is not effective for spatial compatibility, depends on the presupposition that the position with hands held in pronation is somehow a more anatomically "normal" position and may thus define a mapping. This is not completely obvious at first glance, at least not as obvious as the fact that the right hand is on the right side of the body and the left hand is on the left. Thus it may also be pure kinetic differences between the two hand positions that caused the retardation under the palm-up condition. This possibility seems to be supported by the differential effects for fingers obtained between the two conditions, although the present study cannot offer a final solution to this tangential question.

Finally, our results also favor the coding hypothesis over the attentional hypothesis. Since both responding fingers were always either on the right or on the left side of the body midline, we can conclude that it is the position of the responding fingers with respect to each other that is important for the observed effect, and not, as would be required by the attentional hypothesis, their position with respect to the body midline. The attentional hypothesis would be consistent with our findings only by an ad hoc extension that would assume that the tendency to react toward the stimulus source somehow distinguishes between the relative positions of finger responses of one hand on one side of the body midline.

Contrary to our proposal, Katz (1981) explained his compatibility effect for unimanual two-finger choice reactions by Simon's attentional hypothesis, which refers to a natural tendency to respond toward the source of stimulation. This explanation was possible because his data, unlike our results, did not allow for a clear decision between coding and attentional theories. Because in his experimental procedure hands were always held in the prone position in the body midline, the right or left finger position was confounded with the right or left side of the body. Strictly speaking, our findings do not completely refute the attentional hypothesis as an explanation for Katz's results, since our investigation concerned only spatial compatibility with relevant stimulus location. However, in view of the strong similarity of the results for relevant and irrelevant stimulus locations in the case of spatial compatibility for hands (see Umiltà \& Nicoletti, 1985), we may hypothesize that the coding hypothesis is more appropriate than the attentional hypothesis for effects with irrelevant stimulus location as well.

Besides the spatial S-R compatibility effect that we obtained for fingers under both experimental conditions, which is the main outcome of this study, one of our other results offers additional evidence against the attentional and for the coding hypothesis. We did not obtain either overall or in any one of the experimental conditions, an interaction between hand and field; that is, no significant spatial compatibility effect for hands occurred. This is consistent with the coding hypothesis, since for each block of trials the hand used for response was fixed and thus only one hand was involved in the choice reaction. This means that, with respect to responding hands, there was no spatial relationship between two possible choice effectors. The attentional hypothesis, on the contrary, would predict such an additional compatibility effect for hands. Here, the position of the responding hand with respect to the body midline would be effective as a position that is favored by direction of attention if the stimulus occurs on the same side. Similarly, the mapping hypothesis would predict a compatibility effect between hands, because it assumes that the anatomical right and left hand are immediately conceived of as spatially right and left. The neu- 
roanatomical pathway hypothesis would at least predict a low reaction time advantage for compatible field-hand pairings (although, admittedly, the sample may have been too small to detect such a marginal effect).

It should be mentioned that two other studies have obtained results that correspond to an additional compatibility effect for hands. Heister (1986) in a study with lexical decisions and unimanual two-finger-choice reaction, found evidence for spatial compatibility effects for responding hands. Umiltà and Nicoletti (1985, Experiment 3) asked their subjects to react with their right or left hands to red and green stimuli coming from the same position in either the right or left visual field, where the field was fixed for each trial block in the experiment. They obtained a spatial compatibility effect for responding hands, although no relative spatial positions of the stimuli were involved (i.e., here the lack of relative spatial information was on the stimulus, not on the response, side). However, both of these studies investigated spatial compatibility with irrelevant stimulus location, and their results may indicate a still uninvestigated difference between the two types of spatial compatibility.

There is yet another possible explanation for the absence of a spatial compatibility effect for responding hands in our experiment. ${ }^{2}$ We let the hands alternate from block to block. One could speculate that subjects develop strategies relying on features of the task that remain most constant. In the present situation this would mean that a compatibility effect perhaps could occur if the hand used for response were kept fixed throughout the whole experimental session, or at least over more than one block. This could explain the different results mentioned above, because in Heister (1986) the responding hand was the same throughout the experiment, and in Umiltà and Nicoletti (1985, Experiment 3 ) the stimulus position was the same throughout each of the two trial blocks; that is, it was changed only once per experimental session. However, a confirmation of this possible explanation would run counter to the coding hypothesis, which predicts the absence of a hand effect if no choice between hands is required by the experimental condition.

In addition to the S-R compatibility effect, some anatomical differences between index and middle fingers are present in our data. However, these anatomical effects do not influence the spatial compatibility effect investigated in this study, since reaction times for compatible versus incompatible reactions always included reaction times for both index and middle fingers. Besides these finger effects, an overall right-field advantage was obtained. This right-field advantage is well known from other studies of spatial compatibility and may be viewed as expressing either a left-hemispheric specialization for choice reactions (see Efron, 1963) or a general directedness of attention to the right visual field (see Umiltà \& Nicoletti, 1985, and the references therein, pp. 467ff.).

In conclusion, our results with unimanual two-finger choice reactions strongly support the coding hypothesis, implying a genuinely cognitive integration of sensuomotor relationships, and thus contribute to a general theory of spatial S-R compatibility as a cognitive phenomenon.

\section{REFERENCES}

BASHORE, T. R. (1981). Vocal and manual reaction time estimates of interhemispheric transmission time. Psychological Bulletin, 89, 352-368.

Berlucchi, G., Crea, F., Di Stefano, M., \& Tassinari, G. (1977). Influence of spatial stimulus-response compatibility on reaction time of ipsilateral and contralateral hand to lateralized light stimuli. Journal of Experimental Psychology: Human Perception \& Performance, 3, 505-517.

Bradshaw, J. L., \& Perriment, A. D. (1970). Laterality effects and choice reaction time in a unimanual two-finger task. Perception \& Psychophysics, 7, 185-188.

Brebner, J., ShePhard, M., \& CaIRney, P. (1972). Spatial relationships and S-R compatibility. Acta Psychologica, 36, 1-15.

EFroN, R. (1963). The effect of handedness on the perception of simultaneity and temporal order. Brain, 86, 261-284.

FrTTs, P. M., \& SEEGER, C. M. (1953). S-R compatibility: Spatial characteristics of stimulus and response codes. Joumal of Experimental Psychology, 46, 199-210.

Hedge, A., \& MARSh, N. W. A. (1975). The effect of irrelevant spatial correspondences on two-choice response-time. Acta Psychologica, 39, 427-439.

HeisTer, G. (1984). Sex differences and cognitive/motor interference with visual half-field stimulation. Neuropsychologia, 22, 205-214.

HeIsTer, G. (1986). Evidence for stimulus-response compatibility effects in a divided visual field study of cerebral lateralization. Submitted for publication.

Heister, G., \& SCHROEder-Heister, P. (1985). S-R compatibility effect or cerebral laterality effect? Comments on a controversy. Neuropsychologia, 23, 427-430.

Heister, G., \& SCHROEder-Heister, P. (1986). S-R compatibility and frames of reference: A discussion of Ladavas and Moscovitch's (1984) results. Submitted for publication.

KATZ, A. N. (1981). Spatial compatibility effects with hemifield presentation in a unimanual two-finger task. Canadian Journal of Psychology, 35, 63-68.

Ladavas, E., \& Moscovitch, M. (1984). Must egocentric and environmental frames of reference be aligned to produce spatial S-R compatibility effects? Journal of Experimental Psychology: Human Perception \& Performance, 10, 205-215.

Miller, J. (1982). Discrete versus continuous stage models of human information processing: In search of partial output. Journal of Experimental Psychology: Human Perception \& Performance, 8, 273-296.

Nicoletti, R., Anzola, G. P., Luppino, G., Rizzolatti, G., \& UMILTÁ, C. (1982). Spatial compatibility effects on the same side of the body midline. Journal of Experimental Psychology: Human Perception \& Performance, 8, 664-673.

Nicoletti, R., \& UMiLtȦ, C. (1984). Right-left prevalence in spatial compatibility. Perception \& Psychophysics, 35, 333-343.

Nicoletti, R., \& UMILTÀ, C. (1985). Responding with hand and foot: The right/left prevalence in spatial compatibility is still present. Perception \& Psychophysics, 38, 211-216.

Nicoletti, R., Umiltä, C., \& Ladavas, E. (1984). Compatibility due to the coding of the relative position of the effectors. Acta Psychologica, 57, 133-143.

OLDFIELD, R. C. (1971). The assessment and analysis of handedness: The Edinburgh Inventory. Neuropsychologia, 9, 97-113.

Reeve, T. G., \& Proctor, R. W. (1985). Nonmotoric translation processes in the preparation of discrete finger responses: A rebuttal of Miller's (1985) analysis. Journal of Experimental Psychology: Human Perception \& Performance, 11, 234-241.

Simon, J. R. (1969). Reactions toward the source of stimulation. Journal of Experimental Psychology, 81, 174-176.

Simon, J. R., \& Rudell, A. P. (1967). Auditory S-R compatibility: 
The effect of an irrelevant cue on information processing. Journal of Applied Psychology, 51, 300-304.

Umiltí, C., \& NicoletTI, R. (1985). Attention and coding effects in S-R compatibility due to irrelevant spatial cues. In M. I. Posner \& O. S. M. Marin (Eds.), Attention and performance XI (pp. 457-471). Hillsdale, NJ: Erlbaum.

WALLACE, R. J. (1971). S-R compatibility and the idea of a response code. Journal of Experimental Psychology, 88, 354-360.

\section{NOTES}

1. Unlike other authors (e.g., Nicoletti et al., 1982; Umiltà \& Nicoletti, 1985), we prefer to use the term spatial $S$ - $R$ compatibility or (synonymously) spatial compatibility in a wide sense, denoting both relevant and irrelevant stimulus locations. This seems to us to be appropri- ate, since otherwise a convenient general term for both aspects of S-R compatibility for spatial relationships would be lacking. No unified terminology has been established up to now. For example, Katz (1981) used the term spatial compatibility in the same way we do, whereas Nicoletti et al (1982) reserved the term for compatibility effects with relevant stimulus location. Spatial S-R compatibility effects with irrelevant stimulus location are also called Simon effects (this proposal goes back to Hedge \& Marsh, 1975), which, however, does not imply that these effects are explained by Simon's theories (see text below).

2. This possibility was pointed out to us by an anonymous reviewer.

(Manuscript received March 11, 1986; revision accepted for publication July $18,1986$. 